\title{
Intestinal NF-E2-related factor-2 expression and antioxidant activity changes in rats undergoing orthotopic liver autotransplantation
}

\author{
MIAN GE* ${ }^{*}$ XINJIN CHI ${ }^{*}$, AILAN ZHANG, GANGJIAN LUO, GUOLIANG SUN, HANBIN XIE and ZIQING HEI \\ Department of Anesthesiology, The Third Affiliated Hospital of Sun Yat-sen University, \\ Guangzhou, Guangdong 510630, P.R. China
}

Received March 11, 2013; Accepted August 16, 2013

DOI: $10.3892 / \mathrm{ol} .2013 .1576$

\begin{abstract}
Liver transplantation is known to trigger intestinal injuries. Oxidative damage that is induced by reactive oxygen species (ROS) plays a crucial role in ischemia-reperfusion injuries. NF-E2-related factor-2 (Nrf2) and its modulated antioxidant enzymes form the critical endogenous antioxidant system to scavenge ROS. The present study investigated the dynamic changes of intestinal ROS levels, Nrf2 expression and antioxidant enzyme activity following orthotopic liver autotransplantation (OLAT). Sprague-Dawley rats were randomly divided into five groups consisting of one sham group and four groups with rats that underwent OLAT and were evaluated following 4, 8, 16 and $24 \mathrm{~h}$, respectively. The intestinal specimens were collected for histopathological examination and the detection of hydrogen peroxide $\left(\mathrm{H}_{2} \mathrm{O}_{2}\right)$, hydroxyl radical ('OH), malondialdehyde (MDA), reduced glutathione (GSH), superoxide dismutase (SOD) and catalase (CAT) levels and the expression of Nrf2. The present study demonstrated that OLAT resulted in severe intestinal injury, which manifested as a significant change in the intestine pathological scores as early as $4 \mathrm{~h}$ and peaking at $8 \mathrm{~h}$ post-treatment. Oxidative stress was also revealed by the increase of the $\mathrm{H}_{2} \mathrm{O}_{2},{ }^{\circ} \mathrm{OH}$ and MDA levels. Significant decreases were observed in the activity of SOD and CAT and a dramatic decrease occurred in the levels of GSH at 4 and $8 \mathrm{~h}$ post-treatment. All the parameters were restored gradually at 16 and $24 \mathrm{~h}$ post-treatment. The expression of Nrf2 in the intestinal tissues increased significantly at
\end{abstract}

Correspondence to: Professor Ziqing Hei, Department of Anesthesiology, The Third Affiliated Hospital of Sun Yat-sen University, 600 Tianhe Road, Tianhe, Guangzhou, Guangdong 510630, P.R. China

E-mail: heiziqing@sina.com.cn

*Contributed equally

Key words: orthotopic liver transplantation, intestinal injury, oxidative damage, antioxidative enzyme, reactive oxygen species, NF-E2-related factor-2
4, 16 and $24 \mathrm{~h}$ following OLAT. The present study shows that an imbalance between oxidants and antioxidants contributes to intestinal oxidative injury, and that the upregulation of Nrf2 is not sufficient to withstand intestinal oxidative injury following OLAT.

\section{Introduction}

Post-operative multi-organ dysfunction (MODF) and systemic inflammatory reaction syndrome (SIRS) remain the main complications of liver transplantation and are critical problems that contribute to a high mortality rate $(1,2)$. Bacterial translocation and enterogenous endotoxemia following intestinal injury may play a significant role in MODF and SIRS (3). Zheyu and Lunan (4) demonstrated that the motility and barriers of the intestine were destroyed following liver transplantation in rats, as evidenced by the increase in plasma endotoxin levels.

Liver transplantation requires the portal vein to be blocked, causing intestinal congestion, hypoxia in the anhepatic phase and the restoration of blood flow reperfusion, making intestinal ischemia-reperfusion injury inevitable $(4,5)$. Oxidative damage induced by reactive oxygen species (ROS), including the superoxide anion $\left(\mathrm{O}_{2}^{-}\right)$, hydrogen peroxide $\left(\mathrm{H}_{2} \mathrm{O}_{2}\right)$ and the hydroxyl radical ( $\mathrm{OH})$, play a crucial role in the pathogenesis of ischemia-reperfusion injury $(6,7)$. Due to the existence of antioxidative enzymes, including superoxide dismutase (SOD) and catalase (CAT), and antioxidants, including glutathione (GSH), the redox balance is well maintained and the clearance of ROS is promoted (8-10). Several studies have shown that inflammatory mediators, including tumor necrosis factor- $\alpha$ (TNF- $\alpha$ ) and interleukin-1 (IL-1), are involved in the intestinal injury that is induced by liver transplantation $(4,5)$. However, the exact alternative pattern of the oxidant/antioxidant system in the process of intestinal injury following liver transplantation remains obscure.

Nuclear factor NF-E2-related factor-2 (Nrf2) has been demonstrated to regulate the synthesis of antioxidant enzymes and to maintain the antioxidative capacity of the body $(11,12)$. A study using microarrays revealed that Nrf2 regulated the majority of antioxidative enzyme gene expression in the lung (13). Numerous studies have reported that Nrf2 plays a pivotal role in preventing oxidative damage. However, the 
alterations to the expression $\mathrm{Nrf} 2$ remains unclear during an intestinal injury following liver transplantation.

The present study was designed to investigate the dynamic changes in the intestinal ROS levels, Nrf2 expression and antioxidant enzymes activity (SOD, CAT and GSH) occurring $24 \mathrm{~h}$ after orthotopic liver transplantation (OLAT), and to evaluate the imbalance of oxidants and antioxidants.

\section{Materials and methods}

Animals and experimental groups. This study was approved by the Institutional Animal Care and Use Committee of Sun Yat-Sen University (Guangzhou, China) and followed the National Guidelines for the treatment of animals. A total of 35 male rats weighing 220-280 g were randomly assigned into five groups consisting of one sham surgery group (sham; $\mathrm{n}=7)$ and four treatment groups that were tested at $4(\mathrm{n}=7)$, $8(n=7), 16(n=7)$ and $24 \mathrm{~h}(\mathrm{n}=7)$ following OLAT, respectively. Subsequent to being anesthetized, the rats belonging to the sham group were subjected to a laparotomy and vascular dissection, without hepatic vascular exclusion and perfusion. The rats in the other groups underwent OLAT. The intestines, starting from $5 \mathrm{~cm}$ to the terminal ileum, were removed at 4,8 , 16 and $24 \mathrm{~h}$ following OLAT.

Experimental model of rat OLAT. An OLAT model similar to that previously described by Jin et al (14) was applied with certain modifications (15). The rats were fasted for $12 \mathrm{~h}$ and anesthetized using inhaled ether, which was administered through an open face guard. Following the anesthesia, the abdomen was incised, the falciform ligament of the liver was resected and the blood vessels along the esophagus were ligated and disconnected. The suprahepatic vena cava (SVC) was fully liberated and the liver was replaced in its original position. Once the upper region of the left renal vein was completely free, the inferior vena cava (IVC) was dissociated. The first hepatic portal was drawn and the portal vein (PV) was separated from the splenic veins and the convergence of the inferior mesenteric vein. The hepatic artery and the biliary tract were liberated. Subsequently, the portal hepatics were ligated. Microvascular clamps were folded on the convergence of the inferior mesenteric vein, hepatic artery, splenic vein, SVC and IVC. The PV was punctured with a 4th needle in preparation for reperfusion and fixed using a microvascular clamp. Ringer lactate solution (pre-cooled, $4^{\circ} \mathrm{C}$ ) was injected during reperfusion at a speed of $2.5 \mathrm{ml} / \mathrm{min}$ and a $1-\mathrm{mm}$ incision was cut in the wall of the IVC as an outflow tract. The liver color progressively turned yellow if the reperfusion was successful. The recovery of the liver perfusion represented the accomplishment of OLAT. Finally, the needle was extracted and the incision of the PV and IVC was repaired using 8-0 sutures. The clamps on the PV, SVC, IVC and hepatic artery were loosened. The whole anhepatic phase lasted for $20 \pm 1 \mathrm{~min}$.

Histopathological examination. An intestinal segment of $0.5-1.0 \mathrm{~cm}$ in length was cut from $5 \mathrm{~cm}$ to the terminal ileum and fixed in $10 \%$ formaldehyde. Subsequent to being embedded in paraffin, the tissues were stained with hematoxylin and eosin (HE) for light microscopy examination. Intestinal mucosal damage was evaluated and graded according to the following criteria suggested by Chiu et al (16): Grade 0, normal mucosa villi; grade 1, development of subepithelial Gruenhagen's space at the tip of the villi; grade 2, extension of the subepithelial space with moderate epithelial lifting; grade 3, massive epithelial lifting, possibly with a few denuded villi; grade 4, denuded villi with lamina propria and exposed capillaries; and grade 5, disintegration of the lamina propria, ulceration and hemorrhage.

Preparation of specimens and detection of protein concentration in the small intestine. A segment of small intestine was washed with frozen saline, dried with suction paper and frozen at $-80^{\circ} \mathrm{C}$. Intestinal protein levels were measured using a bicinchoninic acid assay (BCA), according to the manufacturer's instructions (Nanjing KeyGen Biotech Co., Ltd., Nanjing, China).

Detection of malondialdehyde (MDA) content. MDA content was measured using the thiobarbituric acid (TBA) method (Nanjing KeyGen Biotech Co., Ltd.). The homogenate $(0.1 \mathrm{ml})$ was used to detect the MDA content. The condensation of MDA and TBA resulted in a red product, which had a maximum absorption peak at $532 \mathrm{~nm}$. The MDA content was calculated by measuring the absorbance at $532 \mathrm{~nm}$ and expressed as $\mathrm{nmol} / \mathrm{mg}$ protein (nmol/mg prot).

Detection of $\mathrm{H}_{2} \mathrm{O}_{2}$ and ${ }^{\circ} \mathrm{OH}$ concentrations. In the acidic environment, $\mathrm{H}_{2} \mathrm{O}_{2}$ is able to oxidize $\mathrm{Fe} 2^{+}$ions to $\mathrm{Fe} 3^{+}$ions, which combine with dye molecules to product $\mathrm{Fe} 3^{+}$dye complexes. This has a maximum absorption wavelength that was proportional to the concentration of $\mathrm{H}_{2} \mathrm{O}_{2}$ at $560 \mathrm{~nm}$. The concentrations of $\mathrm{H}_{2} \mathrm{O}_{2}$ were measured by detecting the absorbance at $560 \mathrm{~nm}$ and were expressed as $\mathrm{mmol} / \mathrm{mgprot}$.

The levels of ${ }^{\circ} \mathrm{OH}$ were determined through the Fenton reaction by detecting the absorbance at $550 \mathrm{~nm}$. The ${ }^{\circ} \mathrm{OH}$ level in the intestinal tissue was expressed as $\mathrm{U} / \mathrm{mg}$ prot.

Detection of SOD and CAT activities and GSH content. The intestinal tissues were homogenized with normal saline solution and centrifuged at $1,500 \mathrm{x} g$ for $15 \mathrm{~min}$ to remove the debris. The supernatant was transferred into fresh tubes for the evaluation of the SOD and CAT activities and GSH content. The measurements were performed using an assay kit (KeyGen Biotech Co., Ltd., Nanjing, China) following the manufacturer's instructions.

SOD activity was determined using the Xanthine oxidase method by detecting the absorbance at $550 \mathrm{~nm}$. SOD activity was expressed as U/mg prot.

CAT was measured by the reaction of CAT scavenging $\mathrm{H}_{2} \mathrm{O}_{2}$. In this reaction, ammonium molybdate was added and a pale yellow complex was produced. The change in the absorbance at $405 \mathrm{~nm}$ was monitored. CAT activity was expressed as $\mathrm{U} / \mathrm{mg}$ prot.

GSH reacted with dithiobis-nitrobenzoic acid to generate 5-dithio-bis2-nitrobenzoic acid dithiobis-nitrobenzoic acid anion, which was a yellow compound and had a maximum absorption peak at $420 \mathrm{~nm}$. The concentrations of GSH were measured by detecting the absorbance at $420 \mathrm{~nm}$ and were expressed as $\mathrm{mg} / \mathrm{gprot}$.

Western blot analysis. Nuclear proteins were extracted from the frozen intestinal tissues using protein extraction kits (KeyGen 
A

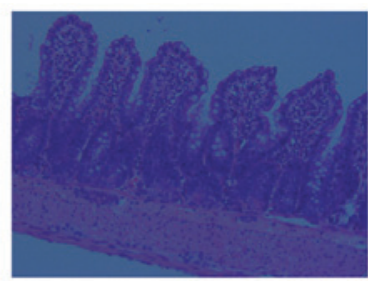

Sham

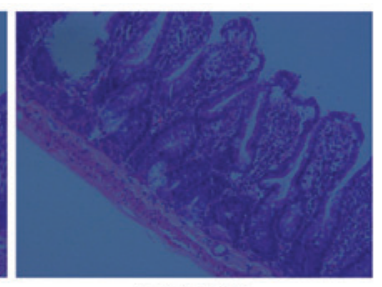

OLAT $4 \mathrm{~h}$

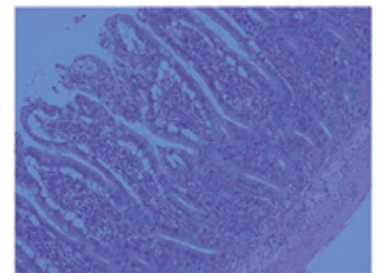

OLAT $8 \mathrm{~h}$

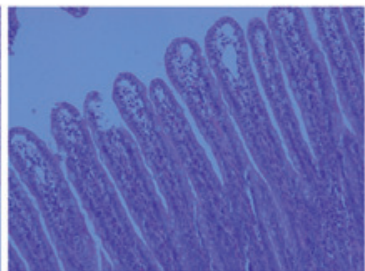

OLAT $16 \mathrm{~h}$

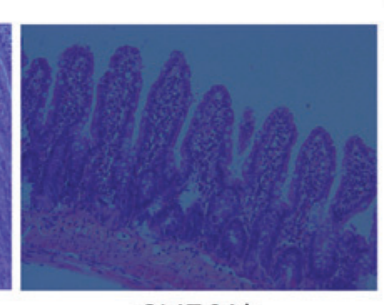

OLAT $24 h$
B

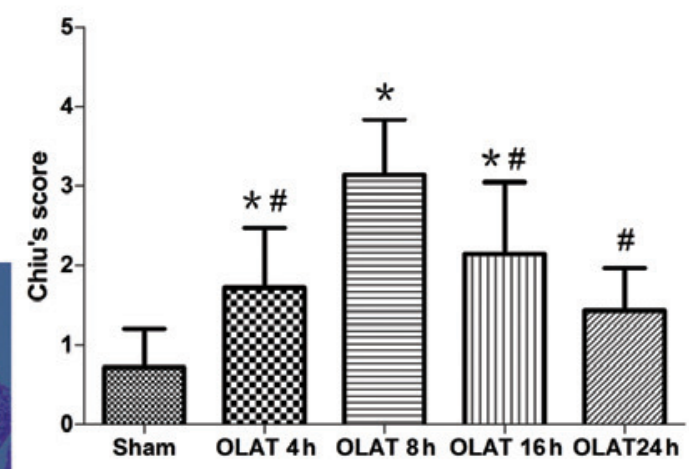

Figure 1. (A) Histological analysis and change in (B) Chiu's score of small intestinal mucosa following HE staining (x200). Results are presented as the mean $\pm \mathrm{SD}$ ( $\mathrm{n}=7 /$ group). ${ }^{*} \mathrm{P}<0.05$ compared with the sham group; ${ }^{*} \mathrm{P}<0.05$ compared with the 8 -h group. OLAT, orthotopic liver autotransplantation.
Biotech Co., Ltd.) for Nrf2 measurements. The protein concentration was measured using the Bradford method.Protein $(60 \mu \mathrm{g})$ was loaded onto a 4-20\% SDS-PAGE pre-made gel (Invitrogen, Carlsbad, CA, USA) for polyacrylamide gel electrophoresis and then transferred to a polyvinylidene fluoride membrane that was pretreated with $100 \%$ methanol. The membranes that were loaded with the protein of interest, Nrf2, were incubated with 5\% skimmed milk. Rabbit monoclonal anti-Nrf2 antibody (dilution, 1:200; Santa Cruz Biotechnology, Inc., Santa Cruz, CA, USA) was added to the supernatant and the mixture was incubated on a rotating wheel at $4^{\circ} \mathrm{C}$ overnight. On the second day, the membranes were washed with TBS three times and incubated with a second antibody conjugated to horseradish peroxidase (dilution, 1:2,000; Santa Cruz Biotechnology, Inc.) for $1 \mathrm{~h}$ at room temperature. Densitometry was used, and normalization to $\beta$-actin immunoreactivity was adopted to correct the differences in the samples.

Statistical analysis. All the data are expressed as the mean \pm SD and were analyzed using SPSS 16.0 software (SPSS, Inc., Chicago, IL, USA). Repeated measurements were applied for the intra-group comparison. $\mathrm{P}<0.05$ was considered to indicate a statistically significant difference.

\section{Results}

Intestinal pathology under light microscopy. No injury was evident in the sham group, which demonstrated normal mucosal villi and glands. However, in the OLAT-induced intestinal injury groups (Fig. 1), the intestinal structure was damaged most severely at $8 \mathrm{~h}$ following OLAT, where almost all the animals demonstrated massive epithelial lifting down the sides of the villi, with a few denuded villi. The intestinal structure was less damaged at 4 and $16 \mathrm{~h}$ following OLAT compared with the animals with a few denuded villi, accompanied with an extension of the sub-epithelial space with moderate or massive epithelial lifting. The Chiu's scores significantly increased at 4,8 and $16 \mathrm{~h}$ following OLAT compared with the sham group $(\mathrm{P}<0.05$ vs. sham group). The Chiu's scores

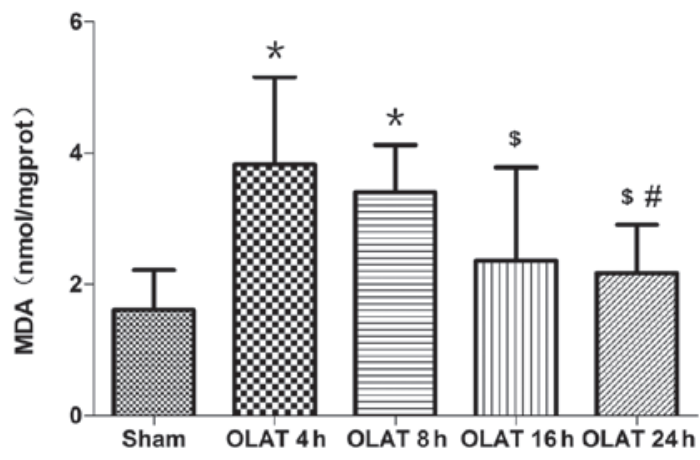

Figure 2. Changes of malondialdehyde (MDA) content in the small intestine Results are presented as the mean $\pm \mathrm{SD}$ ( $\mathrm{n}=7$ /group). ${ }^{*} \mathrm{P}<0.05$ compared with the sham group; ${ }^{\$} \mathrm{P}<0.05$ compared with the 4 -h group; ${ }^{\sharp} \mathrm{P}<0.05$ compared with the 8 -h group. OLAT, orthotopic liver autotransplantation.

peaked at $8 \mathrm{~h}(\mathrm{P}<0.05$ vs. the sham, 4-, 16- and 24-h groups) and then improved gradually (Fig. 1).

MDA content in the small intestine. The content of MDA increased significantly at 4 and $8 \mathrm{~h}$ following OLAT; the content was increased up to $2-2.4$ times $(\mathrm{P}<0.05$ vs. sham group). The MDA content in the 16- and 24-h groups gradually decreased ( $\mathrm{P}<0.05$ vs. 4 -h group; Fig. 2).

$\mathrm{H}_{2} \mathrm{O}_{2}$ content and ${ }^{\circ} \mathrm{OH}$ level in the small intestine. The content of $\mathrm{H}_{2} \mathrm{O}_{2}$ increased by 1.7-1.8 times at 4 and $8 \mathrm{~h}$ following OLAT $\left(\mathrm{P}<0.05\right.$ vs. sham group; Fig. 3). The content of $\mathrm{H}_{2} \mathrm{O}_{2}$ gradually decreased at 16 -and $24-\mathrm{h}$ ( $\mathrm{P}<0.05$ vs. 4 - and 8 -h groups). Compared with the sham group, the levels of ${ }^{\circ} \mathrm{OH}$ increased at 4,8 and $16 \mathrm{~h}$ following OLAT. The increase ranged between 1.6-1.8 times ( $\mathrm{P}<0.05$ vs. sham group). The levels of ${ }^{\circ} \mathrm{OH}$ at $24 \mathrm{~h}$ were lower than those at $8 \mathrm{~h}(\mathrm{P}<0.05$; Fig. 3).

SOD and CAT activity and GSH content in the small intestine. The SOD activity at 4,8 and $16 \mathrm{~h}$ markedly decreased by $20-25 \%$ ( $\mathrm{P}<0.05$ vs. sham group). The SOD activity at $24 \mathrm{~h}$ gradually recovered to the sham group levels (Fig. 4). 

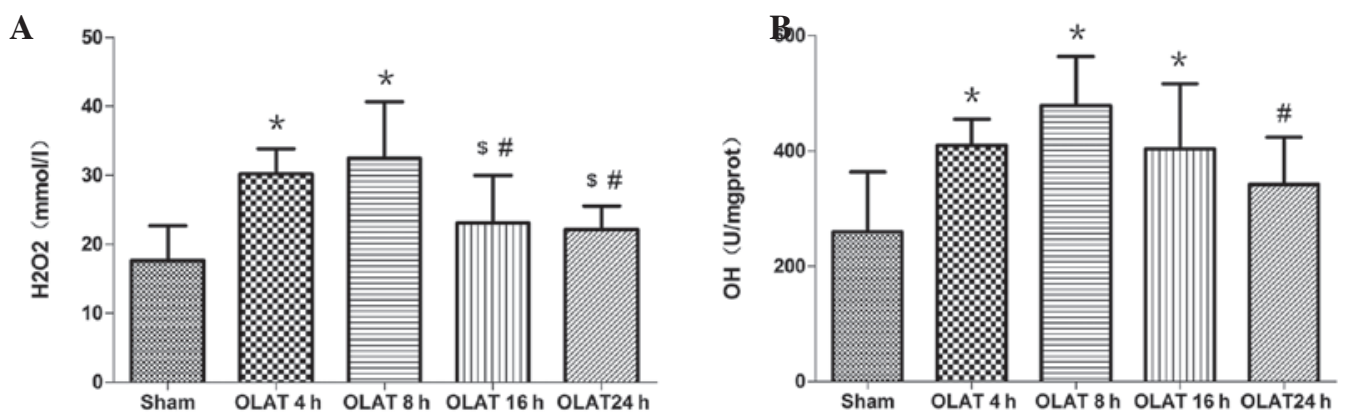

Figure 3. Changes in (A) $\mathrm{H}_{2} \mathrm{O}_{2}$ content and (B) ${ }^{\circ} \mathrm{OH}$ levels in the small intestine. Results are expressed as the mean $\pm \mathrm{SD}$ ( $\mathrm{n}=7 /$ group). ${ }^{*} \mathrm{P}<0.05$ compared with the sham group; ${ }^{\$} \mathrm{P}<0.05$ compared with the 4 -h group; ${ }^{\prime} \mathrm{P}<0.05$ compared with the 8 -h group. OLAT, orthotopic liver autotransplantation.
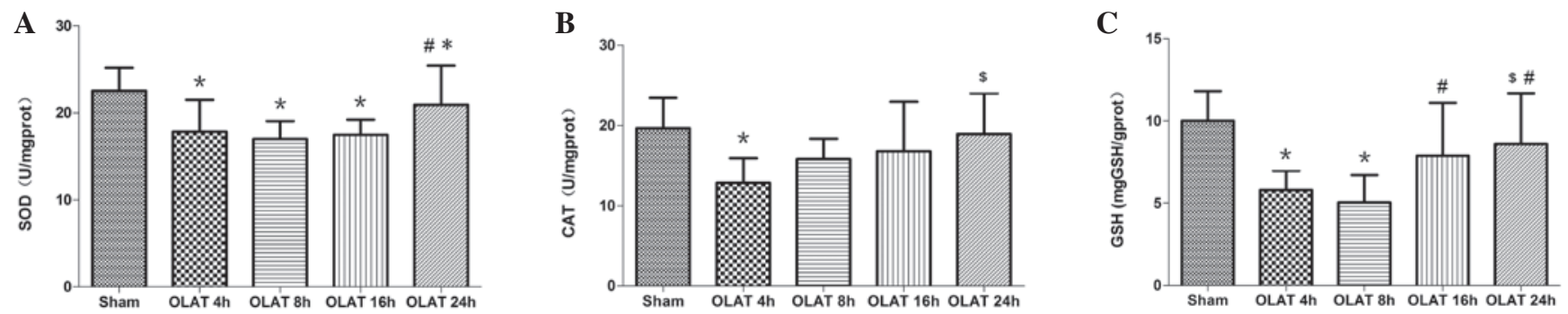

Figure 4. Changes of (A) superoxide dismutase (SOD), (B) catalase (CAT) activity and (C) glutathione (GSH) content in the small intestine. Results are presented as the mean $\pm \mathrm{SD}$ ( $\mathrm{n}=7 /$ group). ${ }^{*} \mathrm{P}<0.05$ compared with the sham group; ${ }^{\mathrm{P}} \mathrm{P}<0.05$ compared with the 4 -h group; $\mathrm{P}<0.05$ compared with the 8 -h group; ${ }^{*} \mathrm{P}<0.05$ compared with the 16-h group. OLAT, orthotopic liver autotransplantation.
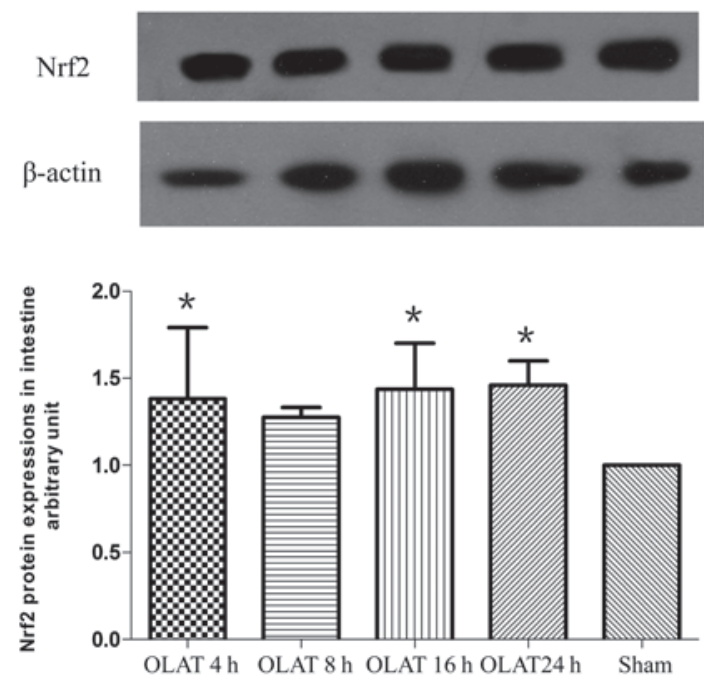

Figure 5. Western blot analysis of the expression of small intestine NF-E2related factor-2 (Nrf2). Results are presented as the mean $\pm \mathrm{SD}$ ( $\mathrm{n}=7$ /group). ${ }^{*} \mathrm{P}<0.05$ compared with the sham group; ${ }^{\mathrm{P}} \mathrm{P}<0.05$ compared with the $4-\mathrm{h}$ group; " $\mathrm{P}<0.05$ compared with the 8 -h group. OLAT, orthotopic liver autotransplantation.

The CAT activity greatly decreased by $\sim 40 \%$ at $4 \mathrm{~h}$ following OLAT (Fig. 4; $\mathrm{P}<0.05$ vs. sham group). The activity of CAT at 8, 16 and $24 \mathrm{~h}$ progressively recuperated the baseline levels ( $\mathrm{P}>0.05$ vs. sham group).

The content of GSH decreased at 4 and $8 \mathrm{~h}$. The decrease was $40-50 \%$ ( $\mathrm{P}<0.05$ vs. sham group). The content of GSH at 16 and $24 \mathrm{~h}$ was higher than the content at 4 and $8 \mathrm{~h}(\mathrm{P}<0.05$; Fig. 4).
Expression of Nrf2 in the small intestine. OLAT induced a $\sim 50 \%$ higher expression of Nrf2 (Fig. 5). Differences were observed at 4, 16 and $24 \mathrm{~h}$ following OLAT ( $\mathrm{P}<0.05$ vs. sham group).

\section{Discussion}

Bacterial translocation and enterogenous endotoxemia following intestinal injury have been known to play a significant role in MODF and SIRS (3). Oxidative damage that is induced by ROS has a crucial role in the pathogenesis of intestinal injury. However the correlation between perioperative intestinal injury and oxidative stress in liver transplantation remains unclear. The present study used the OLAT model, which is able to simulate the key surgical procedures and pathophysiological processes that occur in liver transplantation, including hemodynamic changes, congestion, hypoxia, intestinal reperfusion subsequent to unclamping the portal vein and hepatic ischemia-reperfusion injury (17). The present study identified that OLAT causes serious injury to the pathological structure in the intestines at $8 \mathrm{~h}$ following the procedure. The tissues recover gradually, accompanied with an imbalance in oxidation and antioxidation. The characteristics of the intestinal damage within $24 \mathrm{~h}$ following OLAT were aggravated progressively and then recovered gradually.

Oxidative stress has been known to play a crucial role in organ damage, with $\mathrm{O}_{2}^{-}, \mathrm{H}_{2} \mathrm{O}_{2}$ and ${ }^{\circ} \mathrm{OH}$ being the major ROS (6,7). Antioxidant enzymes and antioxidants, including SOD, CAT and GSH, which constitute the defense system of the body, play a major role in scavenging oxygen free radicals, dealing with oxidative stress and maintaining the redox 
balance (8-10). The increased production of ROS and the decreased production or excessive consumption of antioxidant enzymes or antioxidants may cause an imbalance between oxidants and antioxidants. The MDA content may reflect the degree of lipid peroxidation damage (18). Karabulut et al (19) investigated the changes in the levels of ileum MDA, GSH and nitric oxide (NO) in rats that underwent $45 \mathrm{~min}$ of hepatic ischemia and $30 \mathrm{~min}$ reperfusion, and the results demonstrated that oxidative damage in the ileum was demonstrated by a significant decrease in GSH and an increase in MDA and NO levels. More recently, Tanrikulu et al (20) revealed that hepatic ischemia for $60 \mathrm{~min}$ and a reperfusion time of $90 \mathrm{~min}$ caused histological injury of the intestinal mucosa accompanied by an increase in intestinal MDA and a decrease in GSH-peroxidase. These studies demonstrated that the oxidant and antioxidant imbalance participates in intestinal damage induced by hepatic ischemia-reperfusion.

Hepatic ischemia-reperfusion and intestinal congestion or hypoxia-reperfusion are the main procedures of liver transplantation, but it is uncertain whether or not these procedures change the balance of intestinal oxidants and antioxidants. The present study demonstrated that all the observed oxidative indexes changed most significantly at $8 \mathrm{~h}$ following OLAT, showing that OLAT induced a 1.6-1.8-fold increase in $\mathrm{H}_{2} \mathrm{O}_{2}$ and ${ }^{\circ} \mathrm{OH}$ production, as well as a 2-2.4-fold increase in MDA, a decrease (20-40\%) in SOD and CAT activity and a significant decrease (40-50\%) in GSH. The amplitude of the oxidative and antioxidative indexes decreased at 16 and $24 \mathrm{~h}$ following OLAT. The results of the present study indicated that the imbalance between oxidants and antioxidants contributed to intestinal oxidative injury induced by liver transplantation, particularly in the early phase, while the gradual restoration of the oxidation/antioxidation system balance was beneficial to promoting the repair of the intestine. This investigation indicates that antioxidative intervention is required to alleviate intestinal oxidative injury. Based on the results of the present study, severe complications, including SIRS or MODS following liver transplantation, may be effectively prevented.

Nrf2 is an oxidative-stress sensitive transcription factor. Following the phosphorylation of serine/threonine, stimulated by active oxygen, Nrf 2 combines with antioxidative response element to form a complex that regulates the genetic expression of antioxidase, therefore protecting against oxidative injury by enhancing antioxidant capacity (11-13,21,22). Lu et al (23) used Nrf2-knockout mice and observed that pyrazole caused oxidative liver damage, which was severe in the Nrf2-knockout mice compared with the wild-type mice. Jin et al (24) revealed that Nrf2-deficient mice were more susceptible to traumatic brain injury-induced acute intestinal mucosal injury than wild-type $\mathrm{Nrf}^{+/+}$mice, as characterized by decreased intestinal mRNA expression and antioxidative and detoxifying enzyme activities, including NAD(P)H:quinone acceptor oxidoreductase 1 and glutathione $S$-transferase A1. The present study identified that the expression of intestinal Nrf2 increased at each time point following OLAT, demonstrating that ROS may promote the activation of $\mathrm{Nrf} 2$ and therefore initiate the process of endogenous antioxidation. The extent of alteration to all the parameters at $8 \mathrm{~h}$ following OLAT was further identified; Nrf2 expression was increased $(\sim 40 \%)$, the ROS level was raised $>82 \%$ and the MDA levels were increased by 2.4 -fold.
However, the activity of the antioxidative enzymes decreased. Nrf2 did not offer enough antioxidative enzymes against the intestinal injury that was caused by ROS in the early phase following the graft. However, it may be hypothesized that the intestine may be repaired gradually with an increase of the antioxidase activation regulated by Nrf2.

In conclusion, OLAT results in intestinal damage, which is consistent with the imbalance between oxidation and antioxidation. The upregulation of Nrf2 following OLAT is not sufficient to withstand the oxidative injury in the intestine.

\section{Acknowledgements}

This study was supported by the National Natural Science Foundation of China (NSFC; no. 30972858) and the Planned Science and Technology Project of Guangzhou (no. 2011J4300056).

\section{References}

1. Echániz A, Pita S, Otero A, Suárez F, Gómez M and Guerrero A: Incidence, risk factors and influence on survival of infectious complications in liver transplantation. Enferm Infect Microbiol Clin 21: 224-231, 2003 (In Spanish)

2. Hepp J, Zapata R, Buckel E, et al: General considerations, indications and contraindications for liver transplantation in Chile: a multicenter consensus development document. Rev Med Chil 136: 793-804, 2008 (In Spanish).

3. Swank GM and Deitch EA: Role of the gut in multiple organ failure: bacterial translocation and permeability changes. World J Surg 20: 411-417, 1996.

4. Zheyu C and Lunan Y: Early changes of small intestine function in rats after liver transplantation. Transplant Proc 38: 1564-1568, 2006.

5. Bedirli A, Sakrak O, Soyuer I and Muhtarogul S: Portosystemic shunt prevents apoptosis in rat intestinal mucosa caused by total hepatic ischemia. Eur Surg Res 36: 293-299, 2004.

6. Circu ML and Aw TY: Reactive oxygen species, cellular redox systems and apoptosis. Free Radic Biol Med 48: 749-762, 2010.

7. He SQ, Zhang YH, Venugopal SK, et al: Delivery of antioxidative enzyme genes protects against ischemia/reperfusion-induced liver injury in mice. Liver Transpl 12: 1869-1879, 2006.

8. Matés JM: Effects of antioxidant enzymes in the molecular control of reactive oxygen species toxicology. Toxicology 153: 83-104, 2000

9. Sasaki M and Joh T: Oxidative stress and ischemia-reperfusion injury in gastrointestinal tract and antioxidant, protective agents. J Clin Biochem Nutr 40: 1-12, 2007.

10. Gałecka E, Jacewicz R, Mrowicka M, Florkowski A and Gałecki P: Antioxidative enzymes - structure, properties, functions. Pol Merkur Lekarski 25: 266-268, 2008 (In Polish).

11. Itoh K, Wakabayashi N, Katoh Y, Ishii T, Igarashi K, Engel JD and Yamamoto M: Keap1 represses nuclear activation of antioxidant responsive elements by Nrf2 through binding to the amino-terminal Neh2 domain. Genes Dev 13: 76-86, 1999.

12. Shah ZA, Li RC, Thimmulappa RK, Kensler TW, Yamamoto M, Biswal $S$ and Doré $S$ : Role of reactive oxygen species in modulation of Nrf2 following ischemic reperfusion injury. Neuroscience 147: 53-59, 2007.

13. Cho HY, Reddy SP and Kleeberger SR: Nrf2 defends the lung from oxidative stress. Antioxid Redox Signal 8: 76-87, 2006.

14. Jin C, Zhang PJ, Wu XM, et al: Impact of hypoxic preconditioning on apoptosis and its possible mechanism in orthotopic liver autotransplantation in rats. Hepatobiliary Pancreat Dis Int 8: 40-45, 2009.

15. Chi X, Zhang A, Luo G, et al: Knockdown of myeloid differentiation protein-2 reduces acute lung injury following orthotopic autologous liver transplantation in a rat model. Pulm Pharmacol Ther 26: 380-387, 2013.

16. Chiu CJ, McArdle AH, Brown R, Scott HJ and Gurd FN: Intestinal mucosal lesion in low-flow states. I. A morphological, hemodynamic, and metabolic reappraisal. Arch Surg 101: 478-483, 1970. 
17. Kashfi A, Mehrabi A, Pahlavan PS, et al: A review of various techniques of orthotopic liver transplantation in the rat. Transplant Proc 37: 185-188, 2005.

18. Bela P, Bahl R, Sane AS, Sawant PH, Shah VR, Mishra VV and Trivedi HL: Oxidative stress status: possible guideline for clinical management of critically ill patients. Panminerva Med 43: 27-31, 2001.

19. Karabulut AB, Kirimlioglu V, Kirimlioglu H, Yilmaz S, Isik B and Isikgil O: Protective effects of resveratrol on spleen and ileum in rats subjected to ischemia-reperfusion. Transplant Proc 38: 375-377, 2006.

20. Tanrikulu Y, Kismet K, Serin Kilicoglu S, et al: Diosmin ameliorates intestinal injury induced by hepatic ischemia reperfusion in rats. Bratisl Lek Listy 112: 545-551, 2011.
21. Kaspar JW, Niture SK and Jaiswal AK: Nrf2:INrf2 (Keap1) signaling in oxidative stress. Free Radic Biol Med 47: 1304-1309, 2009.

22. Ishii T, Itoh $\mathrm{K}$, Takahashi S, et al: Transcription factor Nrf2 coordinately regulates a group of oxidative stress-inducible genes in macrophages. J Biol Chem 275: 16023-16029, 2000.

23. Lu YK, Gong PF and Cederbaum AI: Pyrazole induced oxidative liver injury independent of CYP2E1/2A5 induction due to Nrf2 deficiency. Toxicology 252: 9-16, 2008.

24. Jin W, Wang HD, Hu ZG, Yan W, Chen G and Yin HX: Transcription factor Nrf2 plays a pivotal role in protection against traumatic brain injury-induced acute intestinal mucosal injury in mice. J Surg Res 157: 251-260, 2009. 